\title{
Correction to: Evolution at two time frames: ancient structural variants involved in post-glacial divergence of the European plaice (Pleuronectes platessa)
}

\author{
Alan Le Moan (D) Dorte Bekkevold · Jakob Hemmer-Hansen
}

Published online: 29 April 2021

(c) The Author(s), under exclusive licence to The Genetics Society 2021

Correction to: Heredity

https://doi.org/10.1038/s41437-020-00389-3
The original version of this article unfortunately contained mistakes. The presentation of Table 2 and Fig. 4 was incorrect. The corrected Table 2 and Fig. 4 are given below.
Table 2 Summary table of the divergence history between the populations from Iceland and Norway inferred with (SV incl.) and without (SV excl.) the chromosomes carrying putative SVs.

\begin{tabular}{llllllll}
\hline Data & Model & AIC & $\Delta_{\text {AIC }}$ & $W_{\text {AIC }}$ & $T_{S}$ & $T_{\text {SC or AM }}$ & $P$ \\
\hline & SC_af_2m & $\mathbf{1 6 7 8 . 0 0}$ & $\mathbf{0 . 0 0}$ & $\mathbf{0 . 6 7}$ & $\mathbf{5 7 . 9 1}$ & $\mathbf{2 8 . 1 9}$ & $\mathbf{1 . 4 7}$ \\
& SC_af_rf_2m & 1680.00 & 2.00 & 0.25 & 67.46 & 28.26 & 1.60 \\
SVs & IM_af_2m & 1684.00 & 6.00 & 0.03 & 96.40 & - & 49.89 \\
incl. & IM_af_rf_2m & 1684.00 & 6.00 & 0.03 & 96.60 & - & 0.55 \\
& AM_af_2m & 1687.00 & 9.00 & 0.01 & 96.35 & 0.27 & 49.62 \\
& AM_af_rf_2m & 1688.00 & 10.00 & 0.01 & 97.48 & 0.16 & 49.42 \\
\hline & SC_af_2m & $\mathbf{1 6 2 0 . 0 0}$ & $\mathbf{0 . 0 0}$ & $\mathbf{0 . 5 9}$ & $\mathbf{5 4 . 5 6}$ & $\mathbf{2 6 . 7 4}$ & $\mathbf{1 . 0 5}$ \\
& SC_af_rf_2m & 1622.00 & 2.00 & 0.22 & 60.71 & 31.36 & 0.87 \\
SVs & IM_af_rf_2m & 1623.00 & 3.00 & 0.13 & 89.37 & - & 0.44 \\
excl. & IM_af_2m & 1625.00 & 5.00 & 0.05 & 93.68 & - & 49.90 \\
& AM_af_2m & 1628.00 & 8.00 & 0.01 & 92.85 & 0.16 & 47.38 \\
& AM_af_rf_2m & 1630.00 & 10.00 & 0.00 & 96.32 & 0.19 & 48.65 \\
\hline
\end{tabular}

In order of appearance, the table shows the dataset used for the inferences, the six best models sorted by AIC, their AIC, the difference of AIC with the best model $\left(\Delta_{\mathrm{AIC}}\right)$, the weighted AIC $\left(W_{\mathrm{AIC}}\right)$, the time of split between populations $\left(T_{\mathrm{S}}\right)$, the time of secondary contact or ancestral migration $\left(T_{\mathrm{AM} \text { or SC}}\right)$, and the percentage of the genome affected by reduced migration rate $(P)$. The best model for each pairwise comparison is highlighted in bold.

The best model for each pairwise comparison is highlighted in bold. 

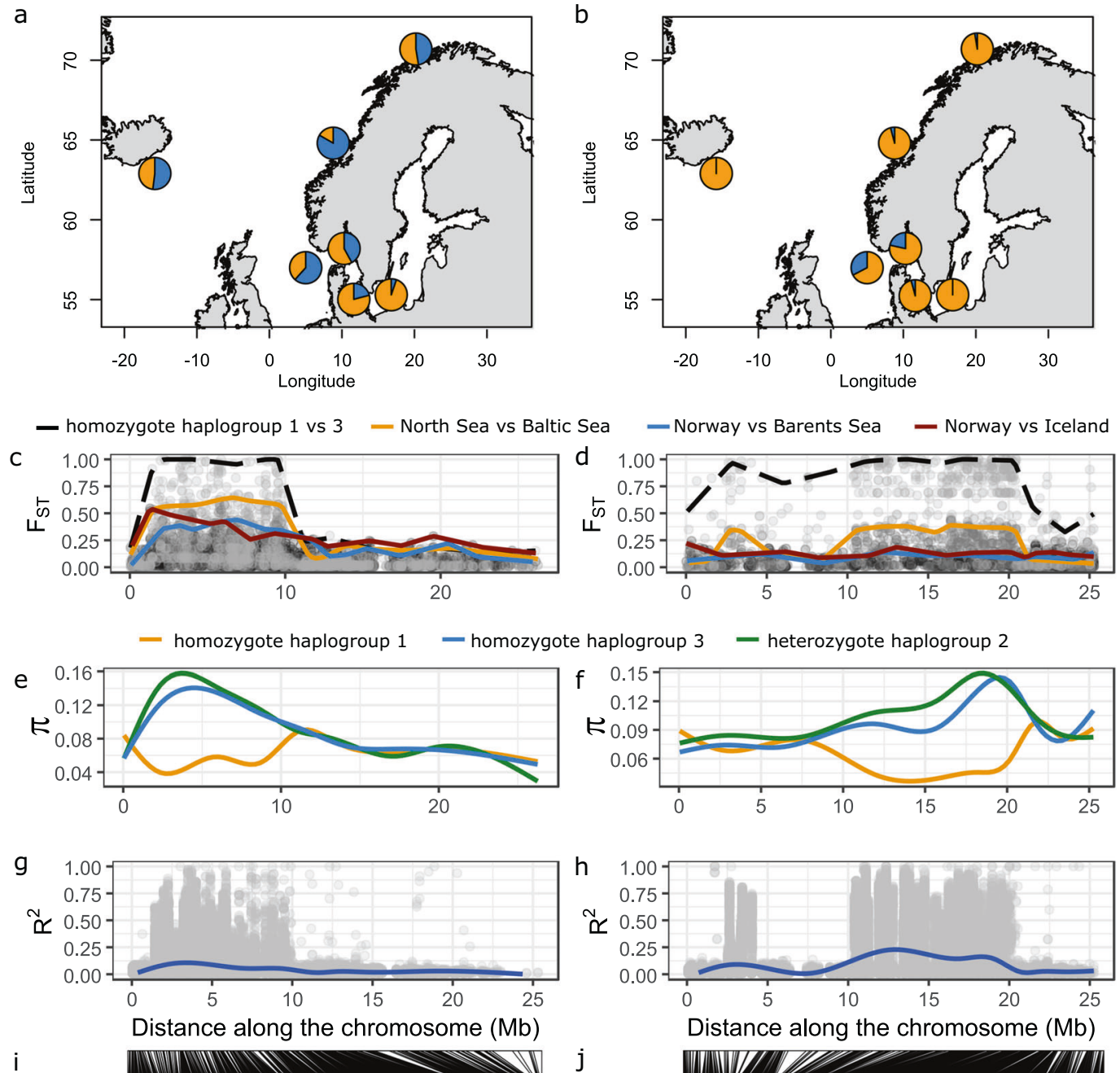

Fig. 4 Detailed analyses of structural variants. Sample-specific data for the European plaice structural variants $\operatorname{SV} 19$ (a, c, e, g, i) and SV21 (b, d, f, h, j): a, b sample-specific allele frequencies (yellow=allele found twice in haplogroup 1 and once in haplogroup 2, and blue $=$ allele found twice in haplogroup 3 and once in haplogroup $2)$; c, d differentiation between different pairs of populations and between haplogroups 1 and 3 along the two chromosomes carrying the SVs, each dot is the FST value of an individual locus and the lines represent the smoothed upper $1 \%$ quantile (one color is one comparison, alpha $=0.3$ ); $\mathbf{e}, \mathbf{f}$ smoothed average $\pi$ for the three haplogroups identified in the DAPC ( $\operatorname{span}=0.3$ ); $\mathbf{g}, \mathbf{h}$ variation of $\mathrm{LD}$ along the chromosomes and $\mathbf{i}, \mathbf{j}$ LD heatmaps for pairwise SNP comparisons. 